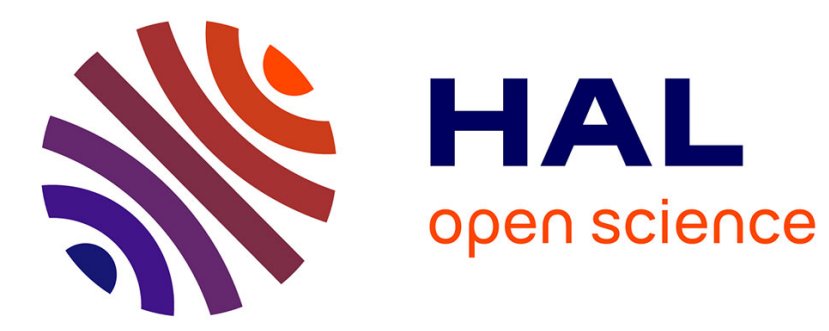

\title{
Les effets de la politique du dépistage organisé du cancer du sein sur l'expérience du travail mammographique des radiologues
}

Béatrice Jacques, Anne-Laurence Penchaud

\section{- To cite this version:}

Béatrice Jacques, Anne-Laurence Penchaud. Les effets de la politique du dépistage organisé du cancer du sein sur l'expérience du travail mammographique des radiologues. Revue française des affaires sociales, 2011, Le métier de médecin, 2-3 (2), pp.210-225. 10.3917/rfas.112.0210 hal-03389887

\section{HAL Id: hal-03389887 \\ https://univ-angers.hal.science/hal-03389887}

Submitted on 21 Oct 2021

HAL is a multi-disciplinary open access archive for the deposit and dissemination of scientific research documents, whether they are published or not. The documents may come from teaching and research institutions in France or abroad, or from public or private research centers.
L'archive ouverte pluridisciplinaire HAL, est destinée au dépôt et à la diffusion de documents scientifiques de niveau recherche, publiés ou non, émanant des établissements d'enseignement et de recherche français ou étrangers, des laboratoires publics ou privés. 


\title{
LES EFFETS DE LA POLITIQUE DU DÉPISTAGE ORGANISÉ DU CANCER DU SEIN SUR L'EXPÉRIENCE DU TRAVAIL MAMMOGRAPHIQUE DES RADIOLOGUES
}

\author{
Béatrice Jacques et Anne-Laurence Penchaud \\ La Doc. française | Revue française des affaires sociales \\ $2011 / 2-n^{\circ} 2-3$ \\ pages 210 à 225
}

ISSN 0035-2985

Article disponible en ligne à l'adresse:

http://www.cairn.info/revue-francaise-des-affaires-sociales-2011-2-page-210.htm

Pour citer cet article :

Jacques Béatrice et Penchaud Anne-Laurence, « Les effets de la politique du dépistage organisé du cancer du sein sur l'expérience du travail mammographique des radiologues »,

Revue française des affaires sociales, $2011 / 2 \mathrm{n}^{\circ} 2-3$, p. 210-225.

Distribution électronique Cairn.info pour La Doc. française.

(c) La Doc. française. Tous droits réservés pour tous pays.

La reproduction ou représentation de cet article, notamment par photocopie, n'est autorisée que dans les limites des conditions générales d'utilisation du site ou, le cas échéant, des conditions générales de la licence souscrite par votre établissement. Toute autre reproduction ou représentation, en tout ou partie, sous quelque forme et de quelque manière que ce soit, est interdite sauf accord préalable et écrit de l'éditeur, en dehors des cas prévus par la législation en vigueur en France. II est précisé que son stockage dans une base de données est également interdit. 


\section{Les effets de la politique du dépistage organisé du cancer du sein sur l'expérience du travail mammographique des radiologues}

Cet article s'intéresse à la profession de radiologue, peu investiguée dans les recherches en sociologie de la santé, et particulièrement aux changements qu'elle a subis depuis la mise en place du dépistage organisé du cancer du sein. Nous souhaitons comprendre comment cette mesure de santé publique est venue bouleverser à la fois le contenu et le sens de la pratique mammographique. Que disent les professionnels de ce programme, qui repose sur un cahier des charges qui vient fortement encadrer leur travail? Remet-il en cause ou non le principe d'autonomie de la profession? Quelles sont les modifications profondes que l'on peut observer dans la façon de réaliser le travail mammographique? Nos résultats s'appuient sur une recherche qualitative qui a utilisé la méthode de l'observation in situ (observations réalisées pendant plusieurs mois dans cinq cabinets de radiologie en Aquitaine) et la méthode de l'entretien semi-directif (cinquante entretiens réalisés auprès de radiologues et de femmes chez qui une anomalie mammographique a été détectée).

\section{Effects of organised breast cancer screening on the mammographic work experience of radiologists}

This article focuses on the radiologist profession, which has scarcely been investigated by health care sociological research, and particularly on the changes it has experienced since the implementation of organised breast cancer screening. How has this public health action modified both the nature and the meaning of mammographic practices? What do professionals say about this programme based on specifications that strictly circumscribe their work? Does it challenge the autonomy of the profession? What deep modifications have been observed in the way they perform their mammographic work? The results achieved by the authors are based on qualitative research using an in situ observation method (observations carried out for several months in 5 radiological units in the Aquitaine region) as well as semi-directive interviews (50 of these were conducted with radiologists and women with a detected anomaly). 


\section{Les effets de la politique du dépistage organisé du cancer du sein sur l'expérience du travail mammographique des radiologues}

Béatrice Jacques et Anne-Laurence Penchaud *

* Béatrice Jacques, maître de conférences en sociologie à l'université Bordeaux-Segalen et chercheure au centre Émile-Durkheim (UMR 5116).

Anne-Laurence Penchaud, sociologue, chargée d'enseignement à l'UFR de sciences médicales de l'université d'Angers et chercheure au LEEST. 


\section{Introduction}

Dès la fin de la Seconde Guerre mondiale, chercheurs et pouvoirs publics vont développer différentes techniques de dépistage du cancer du sein : autopalpation, examen clinique, rayons X, marqueurs sériques, thermographie. Mais seule la mammographie «est parvenue à s'imposer comme test de dépistage pratiqué en routine dans de nombreux pays» (Boissonnat, 2003). Dans les années quatrevingt, l'imagerie du sein, qui avait jusque-là une fonction exclusive de diagnostic, est détournée de son emploi premier pour prendre un rôle de dépistage. Aussi les cabinets de radiologie s'équipent rapidement et la pratique du dépistage mammographique devient routinière au début des années quatre-vingt-dix. Cependant, le dépistage, tel qu'il est réalisé dans les cabinets de radiologie libéraux, est l'objet de critiques (contrôle irrégulier des performances techniques des machines, prescription auprès d’une population culturellement déjà sensibilisée, réalisation d'actes non justifiés...) de la part du monde universitaire qui remet en question son efficacité.

De son côté, l’État, dans sa mission de prévention, œuvre pour un programme de dépistage systématique du cancer du sein avec la sélection d'une population sur des critères épidémiologiques. Cependant, la pratique du dépistage individuel est bien installée dans les cabinets et les essais de l'État pour mettre en place un dépistage de masse sont le plus souvent sans effet. Il faut dire que le projet d'organiser collectivement une pratique médicale se heurte au principe même d'autonomie de la profession (Freidson, 1984). Le médecin est seul maître de sa décision dans le colloque singulier et ne peut se voir contraindre à une pratique et une clientèle. Aussi, pendant de nombreuses années, dépistage individuel et dépistage de masse vont coexister sans que ce dernier puisse s'imposer. C'est seulement en 2001, après de nombreuses résistances de la Fédération des radiologues, que le dépistage du cancer du sein tel que nous le connaissons aujourd'hui en France, comme programme national soumis à des normes très précises, pourra être pleinement mis en place. Pourtant, dès 1992, l'État a souhaité étendre le dépistage organisé à l'ensemble du pays. Les radiologues, comme les pouvoirs locaux ${ }^{1}$, y ont vu non seulement une tentative de récupération de la part de la puissance publique de politiques de santé, qui constituent le plus souvent une vitrine électorale, mais aussi l'imposition d'un contrôle et d'un «encadrement du travail médical» qui n’avaient jamais existé avec une telle ampleur auparavant.

1. En 1994, un conflit oppose la sécurité sociale aux conseils généraux (voir Boissonnat, 2003). 
Si la radiologie fut longtemps réduite à une spécialité complémentaire dans la division du travail médical, désormais, avec une médecine de moins en moins clinique, le radiologue occupe une place centrale dans l'établissement du diagnostic et dans l'orientation de la thérapeutique. Le dépistage organisé du cancer du sein illustre parfaitement l'évolution du métier du radiologue dont le rôle ne se limite plus seulement à la détection de l'anomalie mammographique. Désormais, on attend aussi de lui qu'il organise la prise en charge des femmes jusqu'à l'énoncé du diagnostic. Il n'est plus un simple praticien, il est chargé de mettre en place une mesure phare des politiques de santé publique.

Toutefois, le dépistage organisé du cancer du sein repose sur un cahier des charges strict et donc vient fortement «borner» les pratiques. Pour participer au dispositif, le radiologue doit suivre une formation continue, pratiquer régulièrement un contrôle qualité de son appareillage technique, réaliser un nombre minimal de mammographies dans l'année, produire le diagnostic selon une classification spécifique, ou encore se soumettre à une seconde lecture de ses clichés par un confrère qui confirmera ou non son diagnostic initial. Les exigences du dépistage organisé ne sont pas seulement d'ordre technique, mais aussi d'ordre moral, ce dispositif ne pouvant être efficace et efficient que si les radiologues s'impliquent et incitent les femmes à y participer.

Nous proposons de discuter dans cet article de l'expérience du travail mammographique encadré par ce dispositif de dépistage. Après avoir montré comment les radiologues se représentent ce programme et quels sont pour eux ses apports et ses limites, nous analyserons dans quelle mesure la pratique a dû se modifier pour respecter le protocole imposé par la politique nationale de dépistage. Nous souhaitons ici principalement comprendre pourquoi les radiologues acceptent aussi facilement de participer à ce dépistage organisé qui réglemente fortement leurs pratiques, alors même qu'un tel dispositif de contrôle ne se retrouve dans aucune autre spécialité médicale.

Cet article présente des résultats tirés d'une enquête réalisée pour l'Institut national du cancer, sur l'annonce de l'anomalie mammographique et l'observance des examens complémentaires chez les femmes. D'une durée de deux ans, cette étude répondait à un appel à projet paru en 2007 sur le thème de «l'autonomie des patients et relation des patients au système de soins $»^{2}$.

Cette recherche a privilégié la méthode qualitative. L'observation in situ de consultations mammographiques et des entretiens semi-directifs ont constitué les deux principaux outils d'investigation. L'observation s'est déroulée sur une période de sept mois, dans cinq cabinets de radiologie de Gironde sélectionnés à la suite de l'envoi d'un questionnaire ${ }^{3}$ à l'ensemble des radiologues agréés par la structure départementale de dépistage AGIDÉCA. Cette dernière a mis en place le dépistage organisé du cancer du sein en 2003. En 2009, 92249

2. Pour en savoir plus sur les résultats de l'enquête, voir Jacques et Penchaud (2010).

3. Cette enquête quantitative (avec un taux de réponse de $65,28 \%$ au questionnaire) visait un double objectif : mieux connaître les conditions de réalisation de la mammographie de dépistage et choisir cinq cabinets représentatifs de la pratique pour la phase d'observation. Plusieurs critères ont été retenus : sexe, âge, ancienneté dans la pratique, urbain/rural, nombre de mammographies réalisées par mois, exercice seul ou en groupe, équipement technologique du cabinet (possibilité ou non de réaliser les examens complémentaires dans le cabinet). 
femmes ont été invitées en Gironde. Le taux de participation est au-dessus de la moyenne nationale (57,9 \% contre $53 \%$ en moyenne sur la France). 151 radiologues (soit 72 cabinets) ont réalisé des mammographies de dépistage. Pour 79,8 \% d'entre elles, c'est la technologie numérique qui a été utilisée ${ }^{4}$.

Au total, 265 consultations de dépistage du cancer du sein ont été observées. Cinquante entretiens semi-directifs ont été réalisés auprès de femmes (25) à qui une anomalie mammographique a été annoncée et de radiologues participant au dépistage organisé (25).

\section{Le dépistage au service d'une meilleure image des radiologues}

Un des radiologues rencontrés, qui a commencé à faire des mammographies au début des années soixante-dix, à l'époque de la mise sur le marché des premiers appareils, se souvient des réticences des médecins généralistes de son canton à l'encontre de sa nouvelle pratique. Certains interdisaient même à leurs patientes de faire des mammographies, qu'ils jugeaient inefficaces, servant avant tout les intérêts financiers des radiologues. Au sein de la profession médicale, les radiologues ont toujours eu une image de médecins motivés par l'aspect économique du métier, entretenue par leur niveau de rémunération ${ }^{5}$.

Les radiologues interviewés cherchent à se départir de cette représentation du professionnel attiré par l'argent et, d'une certaine manière, l'acceptation du contrôle des pratiques qu'impose le dépistage organisé constitue un moyen efficace pour lutter contre cette image dépréciative : «C'est pas illégitime qu'on nous demande des comptes. On est quand même des libéraux payés par l'État ${ }^{6} . »$

Les exigences du dépistage organisé sont ici présentées comme la garantie de l'éthique du segment professionnel, permettant d'éliminer les pratiques douteuses et mercantiles. Par exemple, le nombre de mammographies et le recours aux examens complémentaires (échographie, biopsie...) sont désormais mieux encadrés. «Le dépistage nous a fait faire des progrès sur les incidences, on a une rigueur de clichés plus sérieuse, on fait davantage attention à la qualité du cliché, ces contrôles c'est pour ça très utile. Je sais pas si ça va apporter quelque chose en termes de santé publique sur le cancer du sein mais en tout cas sur la pratique des mammos ça a apporté énormément. Les radiologues ont des machines qui sont beaucoup plus performantes, plus surveillées, les manipulateurs sont davantage serrés sur l'incidence. On a tous fait des progrès.» La qualité des clichés, et donc du dépistage, est également améliorée, selon les radiologues seconds lecteurs qui observent un véritable changement

4. AGIDÉCA, Rapport d'activité 2009, disponible sur le site http ://www.agideca.fr/pdf/rapport_activite_2009.pdf 5. La radiologie est la spécialité médicale la plus rémunératrice, avec un revenu moyen issu de l'activité libérale de 202800 euros en 2007. Comparativement, le revenu libéral moyen de l'ensemble des spécialistes pour la même année est de 109400 euros et de 86300 euros pour l'ensemble des médecins libéraux (Fréchou et Guillaumat-Tailliet, 2009).

6. Le questionnaire adressé à tous les radiologues fait apparaître qu'ils réalisent en moyenne un peu plus de 105 mammographies par mois (qu'elles soient ou non au titre du dépistage organisé) : la mammographie est l'un des trois actes qu'ils effectuent le plus fréquemment. 
des pratiques depuis ces dernières années chez certains professionnels qui produisaient jusqu'alors des images qui ne permettaient qu'une interprétation approximative.

En France, le dépistage systématique du cancer du sein a été instauré en 1994 et organisé par département, chacun possédant sa structure de gestion du programme financé par les conseils généraux et les caisses d'assurance maladie. Plusieurs éléments ont favorisé la mise en place de ce dépistage : la prévalence de la pathologie (qui est le premier cancer féminin), son accessibilité à la prévention secondaire (possibilité d'identifier des tumeurs dans une population asymptomatique) et l'efficacité de l'examen mammographique qui est simple à réaliser et peu coûteux.

Le cahier des charges initial de ce programme a été plusieurs fois modifié. Aujourd'hui, il est destiné aux femmes âgées de 50 à 74 ans qui sont invitées à réaliser une mammographie tous les deux ans. Le radiologue doit procéder à un examen clinique et à deux incidences par sein qu'il doit codifier en ACR. La classification ACR, produite par le Collège américain de radiologie, repose sur une valeur prédictive positive (VPP) de cancer de l'image et préconise également la conduite médicale à adopter. Face à des images normales ou bénignes, le radiologue doit classer les clichés en ACR 1 ou ACR 2. Les clichés ACR 3 sont considérés comme des images probablement bénignes, avec une VPP inférieure à $2 \%$. Dans ce cas, le radiologue doit inviter la patiente à un contrôle mammographique dans les mois qui suivent. Le radiologue doit lui proposer un examen histologique lorsqu'il classe les clichés en ACR 4 ou ACR 5 qui renvoient à des images jugées suspectes ou malignes.

Enfin, le cahier des charges prévoit une seconde lecture des clichés : le radiologue adresse les clichés ACR 1 et ACR 2 à la structure de gestion afin qu'un confrère réalise une relecture. Initialement réalisée sur l'ensemble des clichés, la seconde lecture ne concerne plus que les clichés normaux et bénins, pour maximiser le repérage des faux-négatifs. En cas de non-concordance entre les deux lecteurs, la structure de gestion renvoie le dossier au radiologue, accompagné des recommandations du second lecteur. Le radiologue reconvoque la femme pour pratiquer de nouveaux clichés et examens.

\section{Le soutien de la deuxième lecture}

Alors que nous faisions l'hypothèse inverse, la seconde lecture n'est pas appréhendée comme un contrôle des compétences. Elle est principalement décrite comme un moyen de se perfectionner, palliant même parfois le rôle de la formation continue. «J'ai pas l’impression que ça pose problème. [...] Je suis tout à fait partisan de cette deuxième lecture, j'ai toujours été pour,j'ai toujours été partisan du contrôle des connaissances, ça ne me gêne absolument pas»; "j’ai une équipe de manips qui ne supportent pas les retours de l'AGIDÉCA, parce que ça veut dire qu'on n'a pas assez bien travaillé, etc. Je dis non, l'AGIDÉCA pour moi c'est ça : je pense avoir bien fait, et y a un retour, ça veut dire que quelqu'un a vu quelque 
chose que je n'ai peut-être pas vu et qui est peut-être important. Pour moi, c'est ni négatif ni positif, c'est le challenge».

La double lecture est réalisée par un pair qui peut ainsi prendre la place du collègue manquant lorsque l'on souhaite un deuxième avis. En effet, certains radiologues, en particulier ceux qui exercent seuls, s'appuient sur cette lecture pour bénéficier d'un avis complémentaire en cas de doute raisonnable. «Pour aller au fond des choses dans ce truc-là, c'est que de temps en temps, pour être honnête, on s'appuie aussi sur cette deuxième lecture. C'est-à-dire qu'une image qui paraît vraiment bénigne et qu'on se dit je vais faire recontrôler de 3 à 6 mois, non j'ai pas envie d'embêter la patiente ou de refaire le truc, on se dit aussi le deuxième lecteur est vigilant aussi et on peut s'en servir. " On constate ainsi des pratiques de sous-classement des images, consistant à classer en images bénignes (ACR 2), adressées en seconde lecture, des clichés qui correspondent sur plusieurs points à la description de l'ACR 3 (qui ne bénéficie pas de cette relecture et prescrit au radiologue de convoquer à nouveau la femme ultérieurement). Les radiologues parlent ouvertement de ce sous-classement, considérant que le risque lié à cette pratique reste limité si le second lecteur "passe à côté». Ces radiologues, travaillant le plus souvent dans des petits cabinets, ont une patientèle réduite et stable avec qui ils entretiennent une relation de proximité. Ils ont toute confiance en l'observance des femmes, elles se représenteront au cabinet lors de la prochaine convocation de l'AGIDÉCA. Dans la plupart des cas, ils connaissent bien leur histoire pathologique et personnelle qui influe directement sur ce sousclassement. Lors des observations, des radiologues expliquent à l'enquêteur des sous-classements en ACR 2 chez une femme dont on connaît les problèmes personnels pour ne pas «lui causer de tracasseries supplémentaires», ou encore chez une patiente qui a déjà subi plusieurs surveillances qui se sont révélées négatives : "Si je respecte la description ACR c'est un ACR 3 mais je suis à 99,9\% sûre que c'est un kyste donc j'arrête de l'embêter, elle a déjà eu peur. » De façon plus marginale, ce «détournement» de l'objectif initial du principe de double lecture s'observe également chez des radiologues appartenant à des gros cabinets : il est parfois plus simple et rapide de faire appel à la relecture qu'au collègue qui a pris du retard sur ses consultations ou qui travaille à mi-temps dans un autre cabinet.

Cette vigilance du second lecteur joue aussi et surtout comme assurance médico-légale. Pour exemple, lorsque des dossiers reviennent au cabinet pour noncongruence de lecture avec le second radiologue, les professionnels ne cherchent pas à discuter les recommandations émises, préférant les «suivre à la lettre» : «Il me demande de refaire un cliché je le fais, un agrandissement je le fais, je refais ce qu'on me demande. Après si je veux rajouter quelque chose je le fais, si je suis pas d'accord je suis pas d'accord mais je refais exactement ce qu'on me demande.» Ici, la négociation diagnostique n'a pas lieu : l'expertise professionnelle s'efface au profit d'une rationalité, non pas médicale, mais organisationnelle (nous entendons ici la rationalité du dépistage organisé du cancer du sein). Non seulement la seconde lecture fait loi et donc doit être respectée, mais surtout elle sécurise et dégage le professionnel de toutes responsabilités. 
C'est également pour se protéger sur le plan médico-légal que les professionnels veulent faire entrer «toutes» les femmes dans le dépistage organisé : «Ça nous est recommandé très fortement de faire entrer les femmes dans le dépistage organisé et en disant que ça pourrait nous être reproché sur le plan médico-légal, si on le proposait pas. Si elles ne veulent pas, elles sont libres, mais on le marque dans le compte rendu en disant qu'on l'a proposé, qu'elle a refusé.» Dès lors, le dépistage individuel pratiqué par les femmes qui ne souhaitent pas intégrer le dépistage organisé devient une activité plus «dangereuse». Aujourd'hui, les radiologues préfèrent le contrôle des pairs, qui les protège de toute crainte procédurale. Notons que ce tournant dans la pratique concerne une spécialité bien spécifique de la profession médicale, qui réalise d'autres actes également à fort risque judiciaire, telles les échographies obstétricales. Ici, un double mouvement est à l'œuvre : une montée sociale du médico-légal et un encadrement plus fort des pratiques de dépistage. Les radiologues ont bien compris la concordance des événements et se servent de l'un pour maîtriser au mieux l'évolution du premier mouvement.

\section{L'encadrement des pratiques : de la contrainte et des ratés}

La critique du dépistage organisé intervient seulement lorsque le dispositif vient isolément identifier des mauvaises pratiques. La plus importante concerne la production par la structure de gestion de statistiques individuelles du classement ACR qui peuvent donner le sentiment à certains radiologues de se faire «rappeler à l’ordre». Cette pratique touche aux principes auxquels les médecins sont le plus attachés : leur autonomie et leur liberté de diagnostic. Une radiologue parle ainsi de ses propres statistiques concernant ses classements ACR 3 qui sont supérieures à la moyenne des recommandations nationales : «Est-ce que je classe de trop en ACR 3, c'est la question qu'on se pose toujours quand on ouvre la lettre de la structure de dépistage, avec le pourcentage. À chaque fois c'est ça qu'on regarde, est-ce que je suis pas passée à côté d'un cancer? Est-ce que je classe plus en ACR 3 que les autres, peut-être de moins en moins, mais ce que je veux dire c'est que ce n'est pas mon problème, c'est-à-dire que si j'estime qu'une image est classée en ACR 3, c'est que j'estime qu'elle est bénigne mais que je donne la possibilité de surveiller ça sur deux ans $^{7}$. »

Il s'agit à la fois de conserver dans l'espace du colloque singulier son autonomie professionnelle tout en soumettant sa pratique à des programmes de santé publique beaucoup plus larges. On est bien entre autonomie et contrainte, et ce particulièrement quand la structure de dépistage vient montrer du doigt une pratique jugée non conforme. C'est l'effet pervers, la contrepartie de la protection médico-légale.

7. Les structures de gestion sont particulièrement attentives au surdiagnostic en ACR 3 qui génère des examens complémentaires. 
Mais, à bien y regarder, on s'aperçoit que loin de revendiquer plus de liberté qui implique une plus grande responsabilité individuelle, les radiologues souhaitent finalement davantage de contrôle ou plutôt un continuum dans la démarche de contrôle. Les radiologues nous ont particulièrement parlé du problème de l'absence de suivi pour les retours de seconde lecture et pointent ici une des limites du système. Quand le premier lecteur reçoit un retour de seconde lecture, il ne peut plus de nouveau solliciter son avis. Or les radiologues sont unanimes : quand le premier lecteur ne parvient pas à voir ce que le second lecteur considère comme une anomalie, cela aboutit à la production d'un surdiagnostic et donc à un surtraitement: "Si je n'ai pas vu du premier coup quelque chose et qu'on me demande de refaire des clichés par exemple, est-ce que je vais voir l'anomalie, puisque je ne l'ai pas vue une première fois? Quelquefois je ne vois rien, on me redemande un contrôle et je ne sais pas pourquoi. Alors là c'est l'angoisse aiguë, je fais quoi? Je ne peux pas leur renvoyer à l'AGIDÉCA, parce qu'on les renvoie pas. Donc on reste avec notre paquet, la patiente qui est sur les dents car elle est morte de trouille, et de temps en temps je les ai envoyés à X(Centre de lutte contre le cancer), pour avis, et ça a fini en chirurgie trois fois sur quatre, alors qu'il n'y avait rien. »

Un autre radiologue insiste sur les conséquences psychologiques pour les femmes : «À ce moment-là, moi j’ai dit ACR 2, la structure de dépistage dit "non, il y a peut-être quelque chose", alors les patientes elles sont comme ça. Moij'amène toujours le dossier à $X(C L C C)$ et je leur demande un compte rendu écrit. Bon ce n'est pas tous les jours, mais ça m'est déjà arrivé une bonne dizaine de fois. Et ça c'est absolument indispensable d'avoir un avis définitif pour la patiente. Alors on rappelle l'AGIDÉCA et ils disent "non, nous on le revoit pas, de toute façon". La première fois, j'ai téléphoné, j’ai dit "vous plaisantez là". Ça c'est un truc qui ne fonctionne pas bien. » Deux difficultés liées à la politique du dépistage organisé sont ici mises en avant. Alors que dans un premier temps les radiologues peuvent souffrir d'un sentiment d'«ingérence» dans leur pratique, une fois qu'ils l'ont accepté, ils se retrouvent seuls face à la conduite à tenir lors d'un retour de dossier. On leur renvoie finalement la gestion d'une éventuelle erreur de lecture. Deuxièmement, ce type de situation peut être particulièrement stressante pour le professionnel et la patiente et, de fait, amener l'un et l'autre à surinterpréter et investir la présence potentielle d'une anomalie. On voit bien d'ailleurs ici la limite de la protection médico-légale. Car, si le médecin est protégé du fait de n’avoir pas vu ou mal vu, il est de nouveau seul dans son cabinet pour prendre la décision diagnostique.

Tout se passe comme si les radiologues avaient totalement intégré le fait d'être contrôlés dans leurs pratiques. En effet, les différentes contraintes auxquelles leur participation au dépistage organisé les soumet leur paraissent tout à fait «naturelles», justifiées, et ce particulièrement chez les jeunes radiologues qui ont été socialisés au contrôle de la pratique.

Pour les professionnels plus âgés, l'acceptation du contrôle ne s'est pas faite sans heurts. Des radiologues se souviennent de collègues réfractaires lors de la mise en place du dépistage organisé, principalement sur l'aspect de la relecture, vécue comme une mise en cause de leurs compétences. Ces radiologues ont toutefois rapidement pris conscience des bénéfices de cette seconde lecture, qui les protège de la peur de l'erreur diagnostique, en particulier du faux-négatif : «Ça 
n’a pas été facile. Même moi, je me disais, “c'est quoi cet æil de Moscou qui va vérifier que mes clichés sont bons, je suis la meilleure, mes clichés sont excellents, qui c'est qui va dire ça?”. Mais non, très rapidement, non mais c'est un tel luxe pour nous, c'est une pression, moi je vois en dépistage individuel c'est une énorme pression de regarder une femme droit dans les yeux et de lui dire "Madame vous n'avez pas de cancer, vous êtes tranquille pour deux ans". C'est énorme! C'est plus difficile de dire qu'il n'y a rien que d'aller trouver quelque chose. Que ce soit un calcul dans la vésicule ou que ce soit un cancer d'ailleurs, c'est plus cassegueule pour nous de dire "Madame il n'y a rien, au revoir Madame", que l'inverse. Ca c'est le plus difficile en médecine. Donc une deuxième lecture, c'est le luxe. Mais il a fallu comprendre que c'était ça.»

Un autre élément explique cette familiarisation avec le contrôle, à savoir la place du radiologue dans la division du travail médical. Avec l’importance prise par la prescription des examens complémentaires dans l'établissement du diagnostic, et ce dans tous les champs de la médecine, les radiologues se trouvent désormais en première ligne, soumis au regard critique des autres spécialistes (les chirurgiens, par exemple). «Moi je pense que le radiologue, il est habitué à être contrôlé quand même. Il est contrôlé par les cliniciens, par ci, par ça, il est validé, donc moi personnellement je ne me sens pas plus contrôlé là que par le chirurgien qui va opérer une appendicite que j'aurais décrite ou une tumeur que j'aurais décrite et qui va me dire "non, là t’es passé à côté de ce truc ou tu ne l'as pas vu, ou tu t'es gouré j'ai opéré et y avait rien”. » Les radiologues sont dès lors «habitués» au contrôle, qu'il émane des autorités sanitaires ou du reste de la profession. Quels que soient l'examen demandé ou l'organe à expertiser, la pratique du radiologue fait l'objet d'un travail de vérification, d'évaluation critique de la part des pairs, qui nous permet d'avancer l'idée d'une hypersocialisation au contrôle, propre à ce segment professionnel.

\section{Une méconnaissance des controverses}

Si les radiologues rencontrés sont plutôt très favorables au dépistage organisé du cancer du sein, on constate également que ces professionnels ne font jamais référence aux différents travaux qui interrogent les bénéfices médicaux de ce même dépistage. Pourtant, l'histoire du dépistage du cancer du sein, c'est aussi l'histoire de nombreuses controverses qui sont venues et qui viennent encore remettre en question l'efficacité du dépistage organisé par mammographie. Il est important de les présenter succinctement pour comprendre en quoi et comment ces critiques de chercheurs interviennent ou non sur la pratique des radiologues.

En 2003, Bernard Junod et Raymond Massé, dans un article pour la revue Santé publique, partent de deux constats : d'une part, la France a fait du dépistage du cancer du sein une priorité nationale alors que la mortalité par cancer du sein n'a que très peu augmenté (entre 1980 et 2000) et, d'autre part, le dépistage a multiplié les chirurgies considérées comme inutiles. En effet, l'amélioration croissante de la sensibilité des machines permet la détection de cancers du sein qui ne 
sont pas létaux (sans évolution métastatique) et pour lesquels les professionnels de santé proposent malgré tout un traitement (accompagné plus ou moins d'effets délétères). Boissonnat (2004), qui juge les conclusions de Junod et Massé ${ }^{8}$ un peu rapides, montre néanmoins que la mammographie de dépistage a fait l'objet de trop peu de recherches montrant son efficacité. Il insiste sur l'absence de volonté de l'État de se munir d'une instance qui se chargerait d'évaluer la pertinence du programme national de dépistage du cancer du sein et qui pourrait le suspendre ou seulement modifier le test de dépistage choisi. D’autres chercheurs se sont également interrogés sur l'efficience du dépistage du cancer. H.G. Welch, professeur de médecine à l'université de Dartmouth, est le plus connu d'entre eux, avec la traduction en français de son ouvrage au titre polémique : Dois-je me faire tester pour le cancer? Peut-être pas et voici pourquoi. H.G. Welch part de l'idée que le dépistage repose sur deux grands principes : les tests doivent permettre de dépister des cancers à un stade précoce, donc de le traiter rapidement. Or, les études montrent que le dépistage «rate» les cancers d'intervalle qui ont une évolution rapide et qui sont donc les plus mortels. De même, les personnes chez qui on a dépisté un cancer et qui ont donc été traitées rapidement peuvent décéder de la maladie. Pour l'auteur, «il est donc manifeste que le dépistage n'est utile que dans certains cas» (2005). Dans un éditorial pour le New England Journal of Medicine, H.G. Welch (2010) analyse les résultats d'une étude norvégienne et montre que les effets attendus du dépistage (réduire les décès par cancer du sein de 30 \% en dix ans) sont bien moins importants que prévus (la baisse constatée n'est que de $10 \%$ ). L'auteur en conclut, d'une part, qu'il revient aux femmes après avoir été informées des effets délétères du dépistage de décider de réaliser ou non une mammographie, d'autre part, que le dépistage par mammographie «ne devrait plus être considéré comme l'un des indicateurs de qualité d'un système de santé».

Alors que nous aurions pu nous attendre à ce que ces controverses reviennent régulièrement dans le discours des professionnels et que leur existence puisse avoir directement un effet sur leur disposition à respecter les normes imposées, les radiologues que nous avons interrogés ne font que rarement référence aux effets iatrogènes du dépistage organisé, qui n’est présenté que sous ses aspects positifs et ses objectifs initiaux (la baisse de la mortalité par dépistage précoce) : «Si le dépistage a été organisé c'est qu'il avait un intérêt. Je ne me suis vraiment pas posé la question car, encore une fois, depuis que je fais de la radiologie, j'entends parler de ce dépistage du cancer du sein, on m'a toujours dit que c'est un cancer qu'on pouvait diagnostiquer plus tôt par un dépistage précoce et qu'on pouvait le soigner, surtout, donc ça avait un intérêt, à partir de là je vois pas où la controverse existe.»

En fait, peu de radiologues ont connaissance de ces controverses, qui agitent essentiellement la communauté scientifique anglo-saxonne. En France, seuls quelques chercheurs et médecins hospitalo-universitaires s'autorisent un regard

8. Dans un article droit de réponse à V. Boissonnat, B. Junod, R. Massé et C. Quélier (2004) font appel à une étude parue dans le Journal of the National Cancer Institute (Thomas et al., 2002) qui montre que les femmes dépistées ne survivent pas au cancer plus longtemps que les autres femmes dans le cas d'un cancer du sein et qu'il semble même qu'elles décèdent plus des suites des traitements et interventions thérapeutiques (voir également l'annexe de l'article qui propose une traduction du texte "Screening for breast cancer-Summary of evidence" présent sur la base d'information Physician Data Query de l'Institut national du cancer des États-Unis). 
critique. Les radiologues restent fidèles à l'idée à laquelle ils ont été socialisés, à savoir que le dépistage organisé est un progrès, tant sur le plan médical qu'en termes de justice sociale : «Il a changé la pratique de la mammographie d'abord dans le recrutement des femmes puisque je pense que ça a vraiment amélioré la prise de conscience et c'est vraiment un public plus large. Donc le nombre de mammographies a considérablement augmenté en dépistage.» Cette idée est très présente chez les radiologues interviewés même si on sait par ailleurs qu'il perdure des inégalités sociales d'accès au dépistage organisé qui reste moins investi par le public auquel il s'adresse en priorité, à savoir les femmes socialement défavorisées (Duport et al., 2007).

Quelques radiologues, sans faire référence aux controverses ou aux auteurs cités précédemment, abordent néanmoins la question du surdiagnostic que génère le dépistage. Ils observent, en effet, une augmentation des biopsies, notamment pour pallier les erreurs éventuelles de faux-négatifs et les retours de seconde lecture. Et cette surprescription de biopsies n'est pas aussi anodine que peuvent le dire certains de leurs confrères : «On nous dit qu’il faut faire des prélèvements. Après, peut-être que, dans vingt ans, on va nous dire attention parce qu'on a fait trop de prélèvements. Moi j'ai connu ça,j'ai connu la période où on a commencé à prélever, à ponctionner, vraiment partout. Il y a quinze ans. Après il y a eu un signal d'alarme en disant "attention, on fait des prélèvements, des biopsies pour n'importe quoi, donc prudence quand même, y a de la morbidité, y a des complications, y a des hématomes, pour des choses qui sont bénignes, donc attention”, donc on a eu un retour. Là c'est reparti. » Pour les autres, l'augmentation des biopsies est au contraire le signe d'un progrès médical : ces nouveaux examens, prescrits dans un délai très court, rapides, indolores et entraînant rarement des effets secondaires, se substituent aux interventions chirurgicales, unique mode de prélèvement avant leur apparition : «Oui, chaque dépistage a des effets indésirables, c'est-à-dire qu'il y a des tas de femmes qui ont des nodules bénins dans les seins et donc on va faire opérer des femmes qui n'auraient pas été opérées si elles n'avaient pas fait de dépistage sur des lésions bénignes. Alors maintenant moins, on biopsie aussi les microcalcifications, donc ça entraîne moins des prélèvements qui entraînent des inquiétudes. [...] Maintenant, avec les biopsies, on peut éviter l'opération. On opère moins, mais on biopsie beaucoup plus. »

Ces différents extraits d'entretien montrent bien comment les pratiques sont influencées par la production de nouvelles connaissances scientifiques sur l'efficience du dépistage organisé qui produisent de nouvelles recommandations. Mais elles sont peu questionnées par leurs utilisateurs qui, d'une part, se sentent peu légitimes en tant qu'acteur «de terrain» à en faire la critique, et qui, d'autre part, s'accommodent plutôt bien d'une politique de dépistage qui amène une clientèle plus large que l'on peut fidéliser. 


\section{Dépistage individuel et dépistage organisé : pour une homogénéisation des pratiques}

La mise en place du dépistage organisé du cancer du sein dépasse, dès l'origine du projet, le seul objectif de prévention chez une population considérée comme la plus à risque. Il s'agissait, comme l'explique Boissonnat (2004), de faire aussi du dépistage «un outil pédagogique capable à terme d'améliorer plus généralement les pratiques des radiologues coopérant au dépistage de masse». Le dépistage amène de nouvelles normes de pratique qui se fondent dans l'activité quotidienne. Si le ministre Jean-François Mattei, en 2002, permet que la pratique du dépistage individuel puisse parallèlement continuer à exister, l'objectif est bien que le cadre donné au dépistage de masse «institutionnalise» et organise l'ensemble des pratiques de dépistage réalisées dans les cabinets de ville.

En effet, nous avons pu observer un «effet dépistage organisé». Par exemple, la qualité des clichés produits en dépistage individuel a été améliorée grâce au principe de double lecture du dépistage organisé : «L'intérêt aussi de ce dépistage, c'est que ça nous a appris à demander des incidences correctes. C'est-à-dire on se disait "oh, c'est limite...". À partir du moment où on le fait en majorité pour le dépistage organisé, peut-être les manips d'emblée vont se dire “ça ce n'est pas un cliché dépistage...”.» Les exigences du cahier des charges du dépistage organisé, comme l'examen clinique systématique ou encore le nombre de clichés prescrits (deux incidences pour chaque sein), ont aussi été progressivement appliquées au dépistage individuel : "Avant on faisait six clichés, et je me suis calée sur les quatre de la structure de dépistage. Voilà, ça fait un moment que je n'en fais plus six. Moi je fais toujours une échographie, ce qui n'est pas toujours demandé dans le cadre de l'AGIDÉCA, mais je ne la comptabilise pas. »

Toutefois, nous constatons davantage un phénomène d'interpénétration des deux types de dépistage, les modalités de l'un influençant celles de l'autre. Tous les radiologues parlent de l'évolution de leurs pratiques dans le sens d'une homogénéisation entre ces deux dépistages. Les propos de la radiologue précédemment citée montrent comment les pratiques du dépistage individuel débordent sur le dépistage organisé pour lequel l'examen échographique doit resté limité aux cas de seins très denses et difficiles à analyser, en l'absence d'anomalie radiologique ou clinique et d'anomalie sur la mammographie et/ou un examen clinique anormal. D’ailleurs, des enquêtes épidémiologiques mettent en évidence des pratiques d'échographie systématique dans le cadre du dépistage organisé, alors même que le nombre de cancers détectés par la seule échographie est très faible. Pour Brigitte Séradour, l'impact de ces pratiques échographiques n’est pas à négliger, pouvant engendrer une augmentation des faux-positifs et des surveillances (2007).

Cette liberté que s'accordent les radiologues en matière de pratique échographique rejoint le constat de Vincent Boissonnat sur les pratiques déviantes par rapport au script initial du dépistage organisé. D’ailleurs, pour l'auteur, on assisterait moins à une interpénétration des deux types de dépistage qu’à un débordement des pratiques du dépistage individuel vers le dépistage organisé, qu'il explique par le choix, en France, du modèle décentralisé du dépistage organisé. Le syndicat des radiologues, dès les expériences pilotes, a revendiqué une participation active au 
programme de dépistage organisé. Cette aspiration a bien évidemment été entendue par les instances décisionnaires qui avaient conscience que la promotion et la réussite du dépistage organisé ne pouvaient aboutir sans l'investissement de la profession médicale. Aussi, un compromis est-t-il passé entre les «deux parties» pour assouplir les exigences du script originel et les adapter à la radiologie libérale, qui pratique déjà en masse le dépistage individuel (Boissonnat, 2004).

Cette marge de liberté dont disposent les radiologues libéraux ne nous permetelle pas de comprendre leurs opinions si favorables envers le dépistage organisé qui encadre pourtant leurs pratiques? 


\section{Conclusion}

Il est intéressant de noter ici que les radiologues sont le seul groupe professionnel du corps médical à avoir accepté un contrôle des pratiques (par les pairs pour la structure de dépistage) aussi grand. Interrogés sur ce constat, les radiologues rencontrés sont finalement assez peu critiques sur cet encadrement, sur cette normalisation du travail médical pourtant éloignée des valeurs de la profession. Tout se passe comme si leur participation à un objectif de santé publique, leur implication dans un programme collectif, organisé, constituaient désormais les nouvelles normes de régulation de la spécialité. Les professionnels (outre quelques remarques sur la légitimité de certains seconds lecteurs qui ressemblent plus à des règlements de compte locaux) s'accordent à dire que le dispositif du dépistage organisé a été un véritable instrument d'amélioration, de perfectionnement de la pratique mammographique dont bénéficient désormais toutes les femmes (dépistées dans le cadre du dépistage individuel ou du dépistage organisé). Par ailleurs, le dépistage organisé octroie au radiologue un rôle qui ne se limite pas à celui de simple prestataire de la prescription d'un confrère. Dans le dépistage individuel, le radiologue répond à une demande de technique du gynécologue ou du médecin généraliste qui prescrivent le type d'examens à réaliser (mammographie avec ou sans échographie, contrôle échographique seul) et qui organisent la prise en charge lorsque les résultats sont positifs. Dans le dépistage organisé, le radiologue est décideur des examens supplémentaires à entreprendre (pertinence ou non de l'échographie pour compléter la mammographie), est reconnu compétent pour procéder à l'examen clinique, prescrit ou pratique lui-même les examens complémentaires et peut réaliser l'annonce du diagnostic. Ces activités légitiment un rôle actif dans le suivi des patientes qui correspond aux aspirations des radiologues rencontrés et qui sont autant d'éléments qui ont motivé, pour la plupart, le choix de la spécialisation en sénologie. 


\section{Références bibliographiques}

AGIDÉCA (2009), Rapport d'activité, disponible sur le site http://www.agideca.fr/pdf/rapport_activite_2009.pdf

Boissonnat V. (2003), «Un "objet-réseau” : la radiographie de dépistage du cancer du sein», in Cresson G., Drulhe M., Schweyer F.-X. (dir.), Coopérations, conflits et concurrences dans le système de santé, Rennes, ENSP.

Boissonnat V. (2004), «Artefact technique et pouvoir normatif : la mammographie de dépistage des cancers du sein», in Bouchayer F., Cresson G., Schweyer F.-X. (dir.), Normes et valeurs dans le champ de la santé, Rennes, ENSP.

Boissonnat V. (2004), «Fausses évidences et vrais dilemmes en santé publique : le dépistage radiologique des cancers du sein», Santé publique, n 41, p. 9-20.

Duport N., Ancelle-Park R., Boussac-Zarebska M., Uhry Z., Bloch J. (2007), Facteurs d'adhésion au dépistage organisé du cancer du sein : étude FADO-sein, InVS.

Fréchou H., Guillaumat-Tailliet F. (2009), «Les revenus libéraux des médecins en 2006 et $2007 »$, Études et Résultats, DREES, nº 686.

Freidson E. (1984), La profession médicale, Paris, Payot.

Jacques B., Penchaud A.-L. (2010), De l'annonce de l'anomalie à l'annonce du diagnostic: observance, rôle du radiologue et temps de vulnérabilité, rapport final INCa.

Junod B., Massé R. (2003), «Dépistage du cancer du sein et médicalisation en santé publique», Santé publique, n 15 , p. 125-129.

Junod B., Massé R., Quélier C. (2004), «Cancer du sein : danger des certitudes prétendues», Santé publique, $\mathrm{n}^{0} 41$, p. 21-26.

Séradour B. (2007), «Le programme de dépistage français : historique et premier bilan de la généralisation», in Séradour B., Le dépistage du cancer du sein. Un enjeu de santé publique, Paris, Springer.

Thomas D.B, Gao D.L, Ray R.M, Wang W.W, Allison C.J. et al. (2002), "Randomized trial of breast self-examination in Shangai: Final results”, Journal of the National Cancer Institute, vol. 4: 19, p. 1445-57.

Welch H.G. (2005), Dois-je me faire tester pour le cancer? Peut-être pas et voici pourquoi, Canada, PUL.

Welch H.G. (2010), "Screening mammography. A long run for a short slide?", The New England Journal of Medicine, September 23, 363: 13, p. 1276-1278. 